\title{
Estudo da relação entre o movimento circular uniforme e o movimento harmônico simples utilizando a videoanálise de uma roda de bicicleta
}

Study of the relationship between circular uniform movement and simple harmonic movement using video analysis of a bicycle wheel

\author{
E. S. Silva*1
}

${ }^{1}$ Instituto Federal de Educação, Ciência e Tecnologia de Mato Grosso - IFMT, Campus Juína, 78320000, Juína, MT, Brasil.

Recebido em 09 de Maio, 2017. Revisado em 28 de Julho, 2017. Aceito em 31 de Julho, 2017.

\begin{abstract}
Neste artigo são discutidos os resultados de uma videoanálise que permite estudar a relação entre o movimento circular uniforme (MCU) e o movimento harmônico simples (MHS), a partir da filmagem do movimento circular de uma roda de bicicleta. Considerando um ponto $P$ fixo na borda da roda, em movimento circular de raio $R$ e coordenada angular $\varphi$, podemos obter suas componentes cartesianas $x$ e $y$ por meio da videoanálise, observando que elas oscilam ao longo do tempo. Assumindo o movimento de $P$ como sendo um MCU, obtemos analiticamente as coordenadas $x$ e $y$, comparando-as com as coordenadas obtidas pela videoanálise. O modelo permite estudar explicitamente a relação entre MCU e MHS, além de fornecer o raio da bicicleta com precisão de $1 \%$.
\end{abstract}

Palavras-chave: MCU, MHS, Videoanálise, Modelagem matemática.

In this article we discuss the results of a video analysis that allows us to study the relationship between uniform circular motion (UCM) and simple harmonic motion (SHM), from the filming of the circular motion of a bicycle wheel. Considering a fixed point $\mathrm{P}$ at the wheel's edge, in a circular motion of radius $\mathrm{R}$ and angular coordinate $\varphi$, we can obtain its Cartesian components $\mathrm{x}$ and $\mathrm{y}$ by means of video analysis, observing that they oscillate over time. Assuming the motion of $\mathrm{P}$ as an MCU, we obtain analytically the $\mathrm{x}$ and $\mathrm{y}$ coordinates, comparing them with the coordinates obtained by video analysis. The model allows explicit study of the relationship between UCM and SHM, in addition to providing the bicycle radius with accuracy of $1 \%$.

Keywords: UCM, SHM, Video analysis, Mathematical modeling.

\section{Introdução}

A relação entre movimento circular uniforme (MCU) e movimento harmônico simples (MHS) permite a investigação de um movimento a partir do outro. Por exemplo, um planeta orbitando uma estrela em trajetória aproximadamente circular, pode ser visto como um sistema oscilante, dependendo da posição relativa entre o observador e o plano que contém a órbita do objeto celeste. Podemos estimar parâmetros físicos de interesse, considerando a relação entre MCU e MHS, ainda que não sejam claras as informações da orbita circular por observação direta. Em trabalho recente, Cuzinatto, Morais e Souza (2014) utilizaram dados de Galileu Galilei para apontar como este último previu corretamente a dinâmica das órbitas das Luas galileanas em torno de Júpiter [1].

Algumas experiências propostas para estudar a relação entre o MCU e o MHS buscam minimizar a exigência de instrumentos sofisticados [2-4]. Moreira e colaboradores (1991), por exemplo, apresentam um experimento utilizando um disco de raio $R$ e um dispositivo LED.

*Endereço de correspondência: ssf.erick@gmail.com
Na montagem indicada, o disco é acoplado a um motor de toca-discos, que o faz girar com velocidade angular constante. Na extremidade do disco, um LED é fixado. O conjunto é inserido em uma caixa de madeira com uma pequena fenda, no plano de rotação do disco, que permite visualizar o LED. O movimento do LED é visto ao longo de uma linha paralela à fenda, em MHS [4]. Outro dispositivo é descrito em Moysés (1996), utilizando também um toca-discos com um objeto $O$ fixo sobre ele, um pêndulo simples e uma lâmpada. Com a iluminação adequada, a sombra do objeto em movimento circular sobre o toca-discos é vista em uma tela de projeção, em MHS. Na montagem descrita, uma massa pendular é colocada para oscilar ao longo do diâmetro do disco. A projeção do objeto $O$ na tela acompanha a oscilação da massa pendular [3].

$\mathrm{Na}$ verdade, podemos imaginar que um ponto $P$ em MCU possui uma projeção $P_{\mathrm{x}}$ ao longo de um eixo genérico $x$ que contém seu diâmetro. Enquanto o ponto $P$ realiza um movimento circular uniforme ao longo da circunferência $C$, sua projeção $P_{\mathrm{x}}$, realiza um MHS ao longo do diâmetro $D$. 
No presente artigo, apresentamos a videoanálise [5-9] do movimento de uma roda de bicicleta, utilizando esses dados para estudar a relação entre o movimento circular uniforme (MCU) e o movimento harmônico simples (MHS). Inicialmente, obtivemos a evolução temporal da coordenada angular $\varphi$, bem como a distância $R$ do ponto $P$ ao centro da roda, por meio da videoanálise. Em seguida, modelamos o movimento de $P$ como sendo um MCU, o que permite a obtenção analítica das coordenadas $x$ e $y$, considerando a relação matemática existente entre o MCU e o MHS. Finalmente, utilizamos as coordenadas $x$ e $y$ obtidas para determinar o quadrado do raio da bicicleta, a partir da soma em quadratura. Podemos comparar os valores previstos analiticamente com o raio medido para a bicicleta, avaliando se o modelo de MCU admitido para o ponto $P$ é válido.

\section{Estudo da relação entre MCU e MHS por meio da videoanálise}

\subsection{A relação entre $\mathrm{MCU}$ e $\mathrm{MHS}$}

Considere um ponto $P$ em movimento circular uniforme (MCU), no sentido anti-horário, ao longo de uma circunferência de raio $R$. A coordenada $x$ do ponto $P$, em um instante $t$, pode ser obtida a partir do raio $R$ e da coordenada angular $\varphi$ (Fig.1), pois:

$$
x=R \cos \phi .
$$

Observe que a coordenada $x$ localiza o ponto $P_{\mathrm{x}}$, projeção de $P$ sobre o eixo $x$ [2-3]. No entanto, a coordenada angular $\varphi$ pode ser escrita em função do tempo, no MCU:

$$
\phi=\phi_{0}+\omega t
$$

com $\omega$ e $\varphi$ representando, respectivamente, a velocidade angular e a coordenada angular inicial do ponto $P$. Dessa forma, a equação (1) pode ser reescrita na forma:

$$
x=R \cos \left(\phi_{0}+\omega t\right) .
$$

Uma vez obtida a posição $x(t)$ para do ponto $P_{\mathrm{x}}$, podemos obter sua velocidade $v$. Diferenciando a equação $(3) \mathrm{em}$

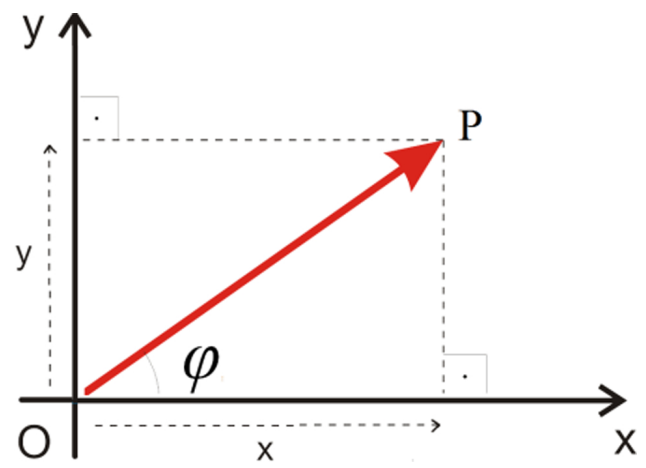

Figura 1: A figura mostra o ponto $P$ formando um ângulo $\varphi$ com o eixo $x$ e suas coordenadas $x$ e $y$. relação ao tempo, temos:

$$
v=-\omega R \sin \left(\phi_{0}+\omega t\right) .
$$

Observe que a velocidade máxima pode ser obtida fazendo:

$$
\sin \phi=(2 n+1) \frac{\pi}{2}, n=0,1,2,3, \ldots
$$

que nos fornece o módulo da velocidade máxima dado por:

$$
\left|v_{\max }\right|=\omega R \text {. }
$$

Analogamente, podemos obter a aceleração do ponto $P_{\mathrm{x}}$. Diferenciando a equação (4) em relação ao tempo, temos:

$$
a=-\omega^{2} R \cos \left(\phi_{0}+\omega t\right),
$$

que pode ser reescrita na forma:

$$
a=-\omega^{2} x
$$

uma vez que $x=R \cos \left(\varphi_{0}+\omega t\right)$. A equação anterior indica que o ponto $P_{\mathrm{x}}$ realiza um MHS de amplitude $R$. De fato, podemos utilizar a segunda lei de Newton e escrever a força $\vec{F}$, sobre o ponto $P_{\mathrm{x}}$, em função da velocidade angular $\omega$ e da coordenada $x$ :

$$
\vec{F}=-m \omega^{2} x \hat{x}
$$

sendo o módulo da aceleração dado por (7) e $\hat{x}$ o vetor unitário na direção $x$. Observe que a expressão 8 pode, ainda, ser escrita na forma:

$$
\vec{F}=-k x \hat{x}
$$

definindo $k=m \omega^{2}$. Isso indica que o movimento do ponto $P_{\mathrm{x}}$ obedece a equação de um oscilador harmônico, de constante elástica $k$ e massa $m[2,3]$.

\subsection{Videoanálise do movimento da roda}

A videoanálise tem sido utilizada para estudar problemas diversos, na literatura nacional e internacional. A referida técnica pode ser definida como sendo a investigação de um fenômeno físico por meio de sua filmagem, considerando a análise desse filme a partir de ferramentas computacionais que permitam o estudo de grandezas físicas relevantes para o seu entendimento [8-11]. As aplicações mais frequentes são em problemas clássicos de cinemática e dinâmica [5-9, 12-16]. Contudo, trabalhos envolvendo física ondulatória, experimento de Millikan, práticas esportivas, entre outras aplicações, também são encontrados na literatura dedicada ao tema [11,17-21].

Um software de relativo sucesso no ensino de física é o Tracker [22]. Trata-se de um programa livre desenvolvido por Douglas Brown, vinculado ao projeto Open Source Physics (OSP) [23]. O programa pode ser utilizado para estudar a evolução no tempo do evento registrado, quadro a quadro, possibilitando a estimativa precisa de parâmetros físicos. 
A única informação requerida pelo programa é o fator de escala, que será utilizado para relacionar as distâncias físicas filmadas com as distâncias correspondentes no vídeo. Por exemplo, fornecendo qualquer distância física situada no plano do movimento observado, o programa realiza o mapeamento das distâncias nos quadros que compõem o vídeo, em pixels. Em nosso caso, inserimos uma distância fixa no quadro da bicicleta, conforme mostra a figura abaixo (Fig.2).

Neste trabalho, utilizamos o Tracker para obter a coordenada angular $\varphi$ em função do tempo, juntamente com a distância $R$ do ponto $P$ ao centro da roda, admitido em MCU. Enquanto o ponto $P$ realiza um MCU, observamos sua projeção no eixo horizontal realizando um MHS. Na Fig.3 ilustramos esse comportamento: o ponto $P$, em vermelho, está em MCU, ao mesmo tempo em que sua projeção $P_{\mathrm{x}}$, em azul, apresenta um MHS ao longo do eixo horizontal (Fig.3). Todo o material utilizado na videoanálise encontra-se em: https://drive.google.com/ open?id\$=\$OBOS3ARHrAIgPMmlKSGpjYWcwWmc.
O mesmo raciocínio pode ser estendido ao movimento de um ponto $P_{\mathrm{y}}$, projeção de $P$ sobre o eixo $y$. De forma análoga, temos:

$$
y=R \sin \left(\phi_{0}+\omega t\right),
$$

com uma lei de força do tipo:

$$
\vec{F}=-k y \hat{y} .
$$

$\mathrm{Na}$ verdade, o raciocínio pode ser estendido a qualquer direção arbitrária (por exemplo, um eixo diagonal qualquer $x_{\mathrm{i}}$ ), que passe pela origem $O$ da circunferência $C$.

\section{Resultados e discussões}

Seja $P$ um ponto fixo na roda da bicicleta filmada, descrevendo uma circunferência de raio $R$ e coordenada angular $\varphi(t)$. O software Tracker permite obter o par ordenado $(\varphi, R)$, que localiza o ponto $P$ ao longo de seu movimento circular. Observe que o raio $R$ pode ser indicado em coordenadas cartesianas $x$ e $y$, admitindo o fator de

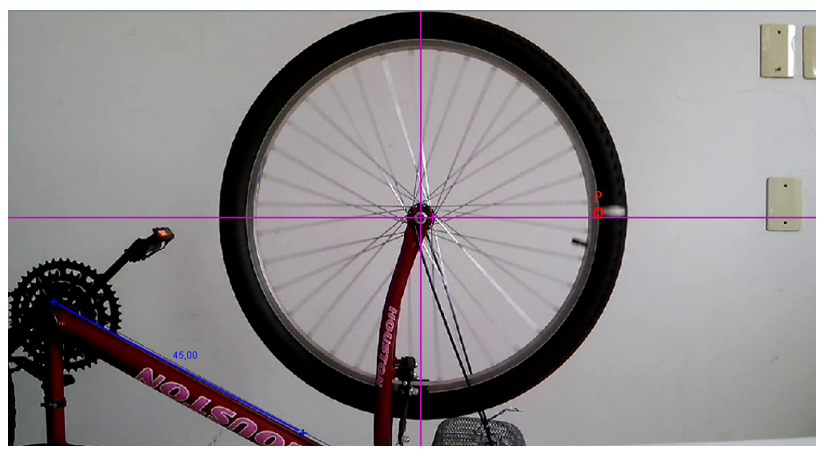

Figura 2: A figura revela o ponto $P$ (em vermelho) estudado juntamente com o fator de escala utilizado, de $45 \mathrm{~cm}$.
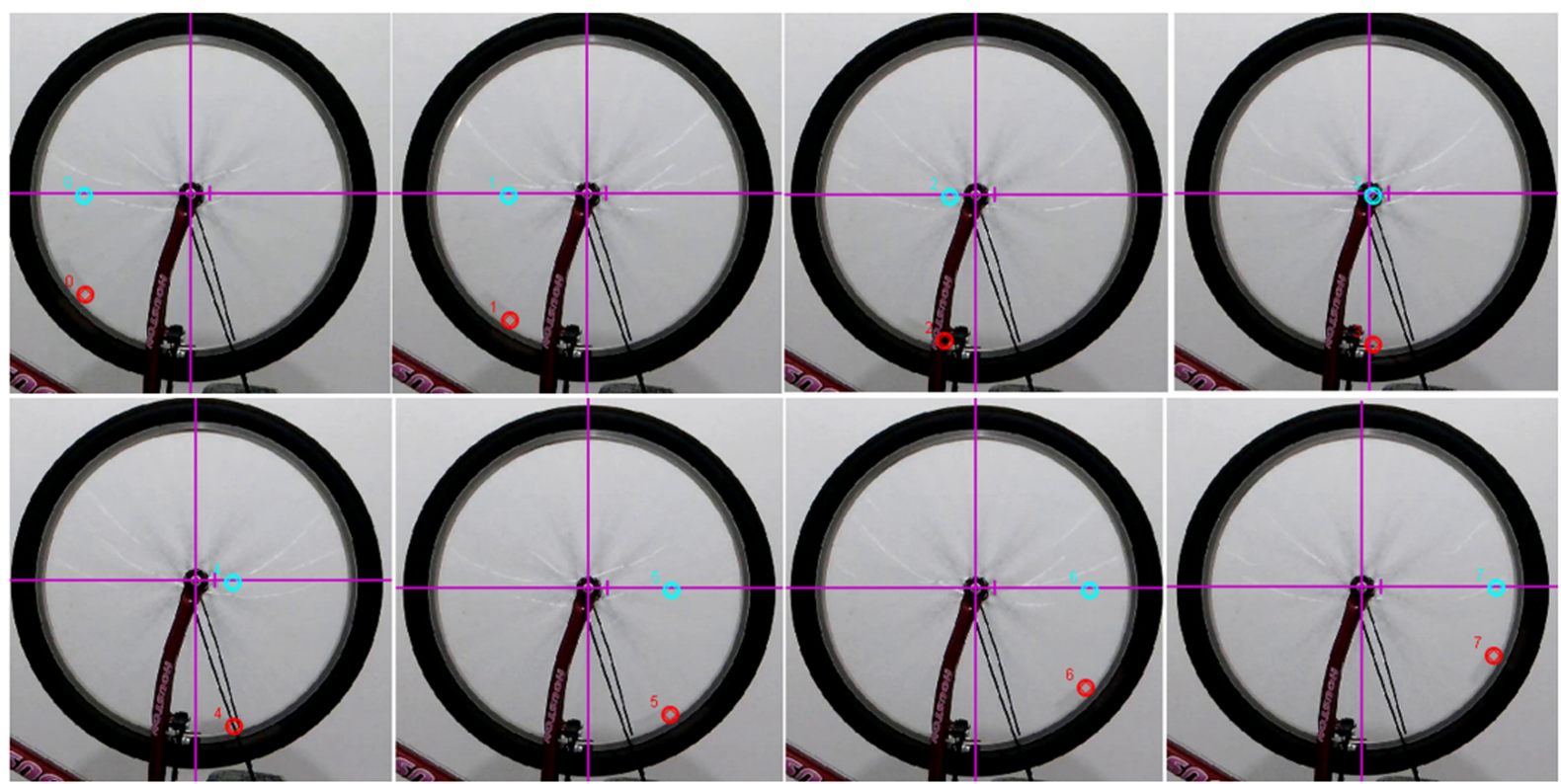

Figura 3: A figura revela o ponto $P$ (em vermelho) descrevendo um movimento circular e sua projeção $P_{\mathrm{x}}$ no eixo horizontal (em azul), descrevendo um movimento oscilatório. A rotação está no sentido anti-horário. 
escala utilizado no quadro. Por exemplo, fornecendo a distância no quadro da bicicleta, igual a $45 \mathrm{~cm}$ (Fig.2), o programa determina as distâncias relativas dos pontos que compõem o vídeo. Isso possibilita, por exemplo, estabelecer a distância entre o centro de rotação e o ponto $P$ na borda da roda. Contudo, a partir das Eq.s (1) e (10), podemos calcular as coordenadas cartesianas analiticamente, a partir do par ordenado $(\varphi, R)$, determinando a equação da trajetória para o ponto $P$. A figura seguinte revela a trajetória do ponto $P$, juntamente com suas coordenadas cartesianas $x(t)$ e $y(t)$, obtidas por meio da videoanálise (Fig.4). Observe que as coordenadas cartesianas de $P$ oscilam no tempo, conforme indicam as Figs.4b e 4c.

A partir das equações (3) e (10), podemos obter as coordenadas analíticas $x^{\prime}$ e $y^{\prime}$ do ponto $P$, comparando-as com as coordenadas $x$ e $y$ fornecidas diretamente pelo Tracker. Considerando o movimento circular de $P$ ao longo de uma volta completa, obtemos desvios absolutos ${ }^{1}$ da ordem de $10^{-8} \mathrm{~cm}$ na referida comparação. Como a precisão do Tracker para as coordenadas cartesianas são da ordem de $10^{-3} \mathrm{~cm}$, podemos observar a coincidência entre as coordenadas analíticas e experimentais, no limite instrumental considerado. Em outras palavras, a comparação mencionada aponta para a validade do modelo que aproxima o movimento de $P$ por um MCU (Tab.1).

Vale observar que no MCU, a coordenada $\varphi(t)$ segue uma relação linear do tipo $\varphi(t)=\varphi_{0}+\omega t$. O ajuste linear dos pontos $(\varphi, t)$ fornece a velocidade angular $\omega$, identificada com o coeficiente angular do ajuste (Fig.5).

Observe que a velocidade angular obtida é igual a $4,16 \pm 0,01 \mathrm{rad} / \mathrm{s}$, ou seja, $238,4 \pm 0,6^{\circ} / \mathrm{s}$. A soma em quadratura das componentes $x$ e $y$ obtidas fornece o quadrado do raio da trajetória $R^{2}$, pois:

$$
R=\sqrt{x^{2}+y^{2}} .
$$

Vale destacar que a equação 12 é justamente a equação da trajetória para o ponto $P$. A comparação dos valo-

${ }^{1} \mathrm{O}$ desvio absoluto $\delta$ é obtido a partir da expressão: $\delta=\left|x-x_{0}\right|$, onde $x$ e $x_{0}$ representam, respectivamente, o valor medido e o valor de referência sobre o qual estamos calculando o desvio [24].
Tabela 1: Desvios absolutos $\delta_{x}$ e $\delta_{y}$ entre as coordenadas cartesianas $x$ e $y$ obtidas na videoanálise e as coordenadas cartesianas $x^{\prime}$ e $y^{\prime}$ obtidas analiticamente, para cada instante de tempo $t$ registrado. O par ordenado $(x, y)$ e $\left(x^{\prime}, y^{\prime}\right)$ são coincidentes no limite experimental.

\begin{tabular}{ccc}
\hline$t(\mathrm{~s})$ & $\delta_{x}(\mathrm{~cm})$ & $\delta_{y}(\mathrm{~cm})$ \\
\hline 0,00 & $3,86 \times 10^{-9}$ & $4,90 \times 10^{-12}$ \\
\hline 0,03 & $3,89 \times 10^{-9}$ & $4,36 \times 10^{-11}$ \\
\hline 0,07 & $3,69 \times 10^{-10}$ & $1,83 \times 10^{-10}$ \\
\hline 0,10 & $6,38 \times 10^{-10}$ & $4,76 \times 10^{-9}$ \\
\hline 0,13 & $3,31 \times 10^{-9}$ & $2,61 \times 10^{-9}$ \\
\hline 0,17 & $1,01 \times 10^{-9}$ & $6,14 \times 10^{-9}$ \\
\hline 0,20 & $2,60 \times 10^{-9}$ & $1,23 \times 10^{-9}$ \\
\hline 0,23 & $4,54 \times 10^{-9}$ & $3,76 \times 10^{-9}$ \\
\hline 0,27 & $1,22 \times 10^{-9}$ & $7,31 \times 10^{-9}$ \\
\hline 0,30 & $2,31 \times 10^{-9}$ & $1,18 \times 10^{-9}$ \\
\hline 0,33 & $3,27 \times 10^{-10}$ & $4,18 \times 10^{-9}$ \\
\hline 0,37 & $2,28 \times 10^{-10}$ & $8,13 \times 10^{-9}$ \\
\hline 0,40 & $7,99 \times 10^{-10}$ & $3,03 \times 10^{-9}$ \\
\hline 0,43 & $1,60 \times 10^{-9}$ & $4,91 \times 10^{-10}$ \\
\hline 0,47 & $1,51 \times 10^{-8}$ & $7,91 \times 10^{-10}$ \\
\hline 0,50 & $1,79 \times 10^{-8}$ & $1,41 \times 10^{-8}$ \\
\hline 0,53 & $2,10 \times 10^{-10}$ & $1,70 \times 10^{-9}$ \\
\hline 0,57 & $6,64 \times 10^{-9}$ & $1,40 \times 10^{-9}$ \\
\hline 0,60 & $1,29 \times 10^{-9}$ & $3,99 \times 10^{-9}$ \\
\hline 0,63 & $2,67 \times 10^{-9}$ & $5,63 \times 10^{-9}$ \\
\hline 0,66 & $1,41 \times 10^{-9}$ & $5,73 \times 10^{-9}$ \\
\hline 0,70 & $3,10 \times 10^{-9}$ & $2,25 \times 10^{-8}$ \\
\hline 0,73 & $3,25 \times 10^{-9}$ & $1,73 \times 10^{-8}$ \\
\hline
\end{tabular}

A precisão fornecida pelo Tracker para as coordenadas cartesianas acima é da ordem de $10^{-3} \mathrm{~cm}$.

res obtidos por meio da videoanálise com o raio $R_{0}=$ $28,20 \mathrm{~cm}$ da bicicleta apresenta desvio percentual em torno de $1 \%$ (Tab.2). Observe que a análise anterior só faz sentido considerando o par ordenado $P(x, y)$, o que evita uma argumentação tautológica (circular).

Vale destacar que, inversamente, podemos obter o ângulo $\varphi(t)$, a partir das coordenadas $x$ e $y$. Seja $x$ uma função na forma:

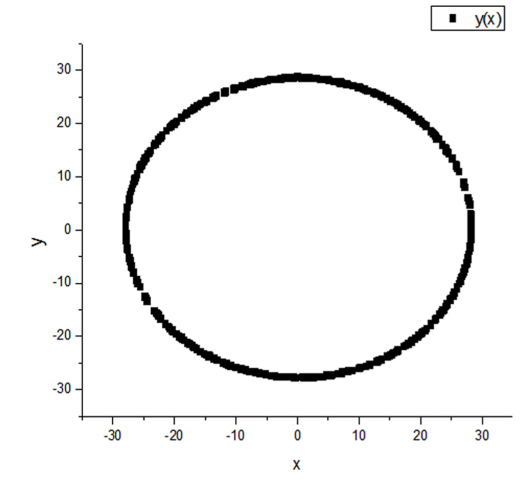

(a)

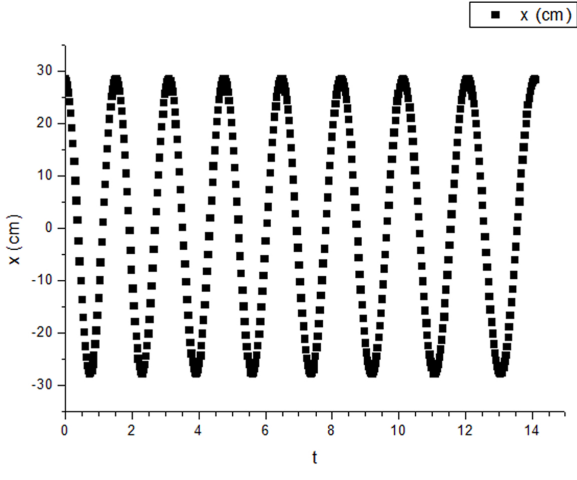

(b)

$$
x=x_{0} \cos (\phi),
$$

$\mathrm{x}(\mathrm{cm})$

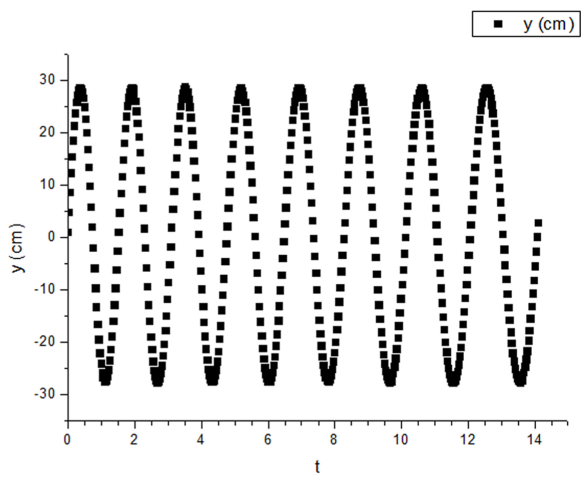

(c)

Figura 4: (a) Trajetória do ponto $P$. (b) Coordenada $x(t)$ do ponto $P$. (c) Coordenada $y(t)$ do ponto $P$. 


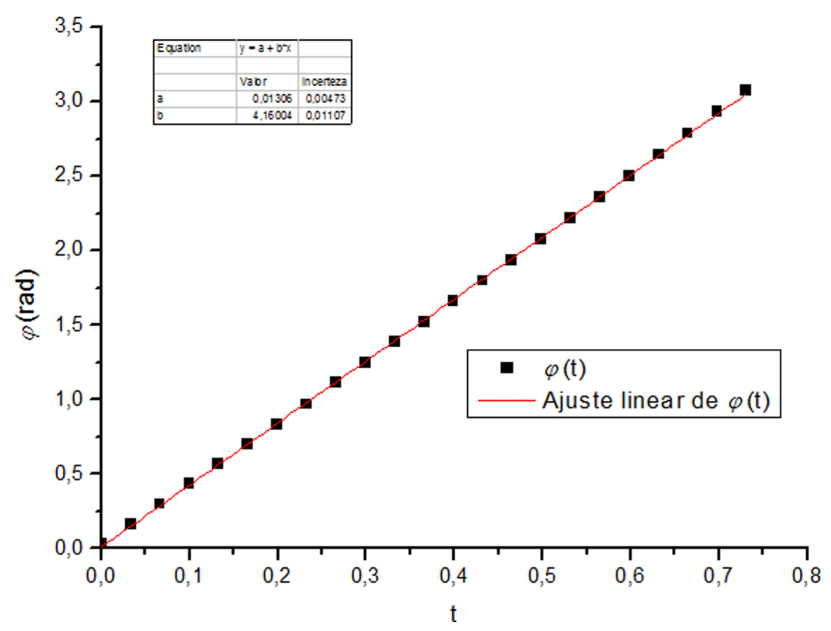

Figura 5: Posição angular $\varphi(t)$ e ajuste linear dos pontos experimentais para uma volta completa de $P$.

Tabela 2: Comparação do raio obtido analiticamente com o raio medido para a bicicleta, igual a $28,20 \mathrm{~cm}$. O instante de tempo $t$ e o erro percentual $\varepsilon$ também são indicados.

\begin{tabular}{lcccc}
\hline$t$ & $x(\mathrm{~cm})$ & $y(\mathrm{~cm})$ & $R(\mathrm{~cm})$ & $\varepsilon \%$ \\
\hline 0,00 & 28,34 & 0,84 & 28,35 & 0,6 \\
\hline 0,03 & 28,07 & 4,70 & 28,46 & 1,0 \\
\hline 0,07 & 27,19 & 8,44 & 28,47 & 1,0 \\
\hline 0,10 & 25,79 & 12,14 & 28,51 & 1,1 \\
\hline 0,13 & 24,06 & 15,30 & 28,51 & 1,1 \\
\hline 0,17 & 21,78 & 18,39 & 28,50 & 1,1 \\
\hline 0,20 & 19,05 & 21,14 & 28,46 & 0,9 \\
\hline 0,23 & 16,10 & 23,55 & 28,53 & 1,2 \\
\hline 0,27 & 12,62 & 25,63 & 28,57 & 1,3 \\
\hline 0,30 & 8,95 & 27,05 & 28,49 & 1,1 \\
\hline 0,33 & 5,18 & 28,00 & 28,47 & 1,0 \\
\hline 0,37 & 1,48 & 28,40 & 28,44 & 0,9 \\
\hline 0,40 & $-2,53$ & 28,22 & 28,34 & 0,5 \\
\hline 0,43 & $-6,43$ & 27,64 & 28,37 & 0,6 \\
\hline 0,47 & $-10,09$ & 26,49 & 28,34 & 0,5 \\
\hline 0,50 & $-13,67$ & 24,73 & 28,25 & 0,2 \\
\hline 0,53 & $-17,03$ & 22,49 & 28,21 & 0,1 \\
\hline 0,57 & $-19,96$ & 19,81 & 28,12 & 0,2 \\
\hline 0,60 & $-22,56$ & 16,70 & 28,07 & 0,5 \\
\hline 0,63 & $-24,68$ & 13,29 & 28,03 & 0,6 \\
\hline 0,66 & $-26,28$ & 9,64 & 27,99 & 0,7 \\
\hline 0,70 & $-27,32$ & 5,74 & 27,91 & 1,0 \\
\hline 0,73 & $-27,86$ & 1,84 & 27,92 & 1,0 \\
\hline
\end{tabular}

O erro percentual $\varepsilon$ (ou desvio percentual) é obtido a partir da expressão: $\varepsilon=\frac{\left|x-x_{0}\right|}{x_{0}} \times 100 \%$, onde $x$ e $x_{0}$ representam, respectivamente, o valor medido e o valor de referência sobre o qual estamos calculando o desvio [24].

que é solução da equação diferencial [25]:

$$
\frac{d^{2} x}{d t^{2}}+\omega^{2} x=0
$$

onde $\omega=\sqrt{\frac{k}{m}}$. A equação descreve um oscilador harmônico de massa $m$ e constante elástica $k$. De fato, de acordo com a segunda lei de Newton, a força resultante é proporcional ao negativo do deslocamento $x$, podendo ser escrita na forma:

$$
m \frac{d^{2} x}{d t^{2}}=-k x
$$

que coincide com a expressão 14 se definirmos $\omega=\sqrt{\frac{k}{m}}$. Observe que a equação 13 pode ser invertida para expressar o ângulo $\varphi$ pretendido, pois $\varphi=\arccos \left(\frac{x}{x_{0}}\right)$.

\section{Considerações finais}

A videoanálise apresentada permite estudar a relação entre o movimento circular uniforme (MCU) e o movimento harmônico simples (MHS), a partir de um arranjo experimental acessível. Verificamos explicitamente que as componentes $x$ e $y$ de um ponto $P$, sobre a roda de uma bicicleta em movimento circular, encontram-se em MHS. Obtivemos uma estimativa da velocidade angular $\omega$ e do raio $R$ da roda. No caso, a comparação do raio obtido pela videoanálise com o raio da bicicleta apresenta erro percentual da ordem de $1 \%$, permitindo apontar para a validade do modelo de MCU, admitido para o ponto $P$.

\section{Referências}

[1] R.R. Cuzinatto, E.M. Morais e C.N. Souza, Revista Brasileira de Ensino de Física 36, 3306 (2014).

[2] D. Halliday, R. Resnick e J. Walker, Fundamentos de Física: Gravitação, Ondas e Termodinâmica (LTC, Rio de Janeiro, 2012), v. 2, p. 87.

[3] H. Moyses Nussenzveig, Curso de Física Basica: Fluidos, Oscilações e Ondas (Edgard Blücher, São Paulo, 1996), v. 2 , p. 39.

[4] S.M.C. Moreira, R.L.N. Pinheiro e L.C. Alvarenga, Caderno Catarinense de Ensino de Física, Florianópolis 8, 227 (1991).

[5] D. Brown and A.J. Cox, The Physics Teacher 47, 145, March (2009).

[6] A.G. Bezerra Jr; L.P. de Oliveira, J.A. Lenz e N. Saavedra, Caderno Brasileiro de Ensino de Física 29, 469 (2012).

[7] J.A. Lenz, N.C. Saavedra Filho e A.G. Bezerra Jr. Abakós, 2, 24 (2014).

[8] V.L.B. de Jesus, Experimentos e Videoanálise - Dinâmica (Livraria da Física, São Paulo, 2014), v. 1, p. 67.

[9] A.G. Bezerra Jr., J.A. Lenz and N.C. Saavedra Filho, Acta Scientiae 18, 782 (2016).

[10] R. Beichner, American Journal of Physics 64, 1272 (1996).

[11] L.I. Leitão, P.F.D. Teixeira y F.S. da Rocha, Revista Electrónica de Investigación em Educación em Ciências 6, 18, 2011.

[12] P. Laws and H. Pfister, Phys. Teach. 36, 282 (1998).

[13] J.A. Monsoriu, M.H. Giménez, J. Riera and A. Vidaurre, Eur. J. Phys. 26, 1149 (2005).

[14] J.A. Bryan, Phys. Educ. 45, 50 (2010).

[15] S. Phommarach, P. Wattanakasiwich and I. Johnston, Physics Education 47, 189 (2012).

[16] P. Klein, S. Gröber, J. Kuhn and A. Müller, Physics Education 49, 37 (2014). 
[17] J. Bonato, L.M. Gratton, P. Onorato and S. Oss, Phys. Educ. 52, 045017 (2017).

[18] A.G. Bezerra, J.A. Lenz, N.C. Saavedra Filho, M.V. Peres, O. Cossi, A.C. Mello and S.A.H. Conceição, Acta Scientiae 17, 813 (2015).

[19] O. Pinto Neto, M. Magini e M.M.F. Saba, Revista Brasileira de Ensino de Física 28, 235 (2006).

[20] D.F. Collins, Phys Teach 38, 561 (2000).

[21] R. Salmon, C. Robbins and H. Forinash, Eur. J. Phys. 23, 249 (2002).

[22] http://physlets.org/tracker, acessado em 06/05/2017.

[23] http://www.opensourcephysics.org acessado em $25 / 07 / 2017$.

[24] J.H. Vuolo, Fundamentos da Teoria de Erros (Edgard Blücher, São Paulo, 1996), v. 1, p. 53.

[25] W.E. Boyce e R.C. Diprima, Equações Diferenciais Elementares e Problemas de Valores de Contorno (Ed. Guanabara Dois, Rio de Janeiro, 1979), v. 1, p. 105. 